\title{
Interfacial room temperature magnetism and enhanced magnetocaloric effect in strained $\mathrm{La}_{0.66} \mathrm{Ca}_{0.34} \mathrm{MnO}_{3} / \mathrm{BaTiO}_{3}$ heterostructures
}

\author{
N. S. Bingham, ${ }^{1,2,{ }^{*}}$ A. K. Suszka,,${ }^{1,2,}$ C. A. F. Vaz, ${ }^{3}$ H. Kim, ${ }^{4}$ and L. J. Heyderman ${ }^{1,2}$ \\ ${ }^{1}$ Laboratory for Mesoscopic Systems, Department of Materials, ETH Zürich, 8093 Zürich, Switzerland \\ ${ }^{2}$ Laboratory for Micro and Nano Technology, Paul Scherrer Institut, 5232 Villigen PSI, Switzerland \\ ${ }^{3}$ Swiss Light Source, Paul Scherrer Institut, 5232 Villigen PSI, Switzerland \\ ${ }^{4}$ Naval Research Laboratory, Washington, DC 20375, USA
}

(Received 25 May 2016; revised manuscript received 31 May 2017; published 13 July 2017)

\begin{abstract}
We present a modification of the magnetization and an electronic reconfiguration due to interfacial coupling between strain relaxed $\mathrm{La}_{0.66} \mathrm{Ca}_{0.34} \mathrm{MnO}_{3}$ and ultrathin $\mathrm{BaTiO}_{3}$ films using the magnetocaloric effect and photoemission electron microscopy at the $\mathrm{Mn}$, Ti $L_{2,3}$ edges. The addition of a top $\mathrm{BaTiO}_{3}$ layer leads to strain-induced enhancement of the magnetocaloric effect in $\mathrm{La}_{0.66} \mathrm{Ca}_{0.34} \mathrm{MnO}_{3}$, due to the structural transitions in $\mathrm{BaTiO}_{3}$, and to room temperature ferromagnetism due to electronic reconfiguration at the interface.
\end{abstract}

DOI: 10.1103/PhysRevB.96.024419

\section{INTRODUCTION}

The venerable perovskite manganites $\left(\mathrm{R}_{1-x} \mathrm{~A}_{x} \mathrm{MnO}_{3}\right)$ have generated immense interest since their discovery in the 1950s $[1,2]$ due to the complex interplay between competing magnetic, electronic, and structural degrees of freedom. Such interplay leads to interesting phenomena such as colossal magnetoresistance (CMR) [3], multiferroicity [4], charge ordering $[5,6]$, and a variety of magnetic properties [7]. Zener's double exchange model was initially proposed [8] in order to explain these order parameters, where $\mathrm{Mn}^{3+}$ and $\mathrm{Mn}^{4+}$ ions exchange charge carriers via the oxygen $2 p$ orbitals. However, this theory fails to accurately predict the role of the lattice, as well as CMR behavior [9-12]. In order to obtain a better understanding, the role of strong electron-phonon coupling (such as lattice polarons and Jahn-Teller distortion [13]) must be considered.

Recently [14,15], $\mathrm{La}_{0.66} \mathrm{Ca}_{0.34} \mathrm{MnO}_{3} \quad$ (LCMO) and $\mathrm{La}_{0.66} \mathrm{Sr}_{0.34} \mathrm{MnO}_{3}$ thin films grown on $\mathrm{BaTiO}_{3}$ (BTO) substrates have been shown to exhibit large jumps in temperaturedependent magnetization and giant magnetocaloric effect (MCE), due to induced strain from first-order structural phase transitions, where BTO changes from rhombohedral to orthorhombic at $183 \mathrm{~K}$, and from orthorhombic to tetragonal at $278 \mathrm{~K}$ [16]. This modification is magnified in the LCMO film due to the similarity between the $\mathrm{La}$ and $\mathrm{Ca}$ ions, which makes LCMO prone to exhibit mesoscopic phase separation due to $\mathrm{MnO}_{6}$ tilting when subjected to external perturbations. Generally, many of the studies on manganite interfaces involve substrate-induced strain on the magnetic film [17-21]. This results in changes of the film properties with the film adjusting to the in-plane symmetry of the substrate. However, little work has been dedicated to the role of interfacial effects on the bulk film properties.

Interfaces of thin film manganite heterostructures are well-documented sites for fundamentally altered magnetism $[22,23]$. Superlattices of ferromagnetic and antiferromagnetic

\footnotetext{
*binghamn@gmail.com; Currently a National Research Council Research Associate at the U.S. Naval Research Laboratory, Washington, DC 20375, USA.

†anna.suszka@psi.ch
}

layers can lead to an enhancement of the total magnetization through an induced ferromagnetic ordering extending into the otherwise antiferromagnetic layer via charge carrier transfer [24], while ferromagnetism has also been observed at the interfaces between two nominally insulating antiferromagnets [25]. The control of the physical properties of the manganites through charge modulation at the interface with other materials alters the crystal field at the interface of the manganite, thus creating new interactions via tilting of the $\mathrm{Mn}-\mathrm{O}$ bond angles or hybridization with a nearest (next-nearest) neighbor and an overall redistribution of localized Mn charge.

In these strongly correlated systems, slight changes in the electronic density can modify the overall balance between competing ground states and, in turn, reveal new states not accessible in the bulk [26]. For example, it is predicted that weakly strained films can form nanoscale magnetically and/or electronically ordered clusters [27], two-dimensional electron gases, and superconductivity [28]. While the consequences of interfacial magnetism on the transport properties have been studied in great detail $[22,29,30]$, a clear microscopic understanding of its impact is still lacking due to challenges inherent to the characterization of interfaces.

We report a combined charge carrier transfer and structurally induced modification of the magnetic and electronic properties of an LCMO film by utilizing the structural transitions of a coupled epitaxial BTO layer. The BTO film thickness $(5 \mathrm{~nm})$ is chosen such that the tetragonal to orthorhombic phase transition coincides with the magnetic phase transition of LCMO, at $250 \mathrm{~K}$ [16]. We show that in such a system, there is a strong enhancement of the magnetocaloric properties in addition to a persistence of interfacial magnetic order at room temperature, which we relate to charge modification at the BTO-LCMO interface. Additionally, such a thin BTO capping layer provides an excellent sample geometry for $\mathrm{x}$-ray photoemission electron microscopy (XPEEM) studies of the LCMO/BTO interface due to an electron escape depth of 5-7 nm.

\section{METHODS}

In order to fully characterize the macroscopic physical properties of the film, we employ MCE, which describes the 
adiabatic temperature (isothermal entropy) change due to magnetizing a magnetic material, an effect that has been explored for magnetic refrigeration applications [31]. MCE has also been proven to be a sensitive probe for complex phase transitions [32-34]. However, the calculated absolute value of the magnetic entropy change $\left(\Delta S_{M}\right)$ becomes ambiguous at firstorder phase transitions, due to the discontinuity in the order parameter. Also detrimental from an application point of view are heat losses due to thermal and magnetic hysteresis associated with first-order transitions [33]. Nevertheless, much information can be gained about magnetic materials using MCE measurements. Since the isothermal MCE is dependent on the derivative of the magnetization, it is inherently more sensitive to small changes in the magnetic properties than standard magnetometry methods. Additionally, one can use the information produced from MCE measurements to determine the order of a transition [35]. MCE is determined by measuring isothermal magnetization $(M)$ versus applied field $(H)$ curves and integrating between the curves using the thermodynamic Maxwell relation to achieve the overall change in magnetic entropy:

$$
\Delta S_{M}=\mu_{0} \int\left(\frac{\partial M}{\partial T}\right)_{H} d H,
$$

where $\mu_{0}$ is the vacuum permeability.

LCMO and BTO films were deposited on $\mathrm{MgO}$ (100) substrates via pulsed-laser deposition (PLD) using a pulsed excimer laser $(248 \mathrm{~nm}$, pulse width $=20 \mathrm{~ns})$, with commercially obtained polycrystalline targets, at a fixed targetsubstrate distance of $6 \mathrm{~cm}$ and laser fluence of $2 \mathrm{~J} / \mathrm{cm}^{2}$. The substrate temperature during deposition was $1050 \mathrm{~K}$ with an $\mathrm{O}_{2}$ partial pressure of 400 mTorr during deposition. After deposition, the films were annealed at $1050 \mathrm{~K}$ for $2 \mathrm{~h}$ in 380 Torr $\mathrm{O}_{2}$ and cooled to room temperature at $5 \mathrm{~K} / \mathrm{min}$. The nominal film thickness of LCMO and BTO films is 50 and $5 \mathrm{~nm}$, respectively, confirmed with atomic force and scanning electron microscopy measurements. The choice of $\mathrm{MgO}$ substrates ensures a fully strain-relaxed LCMO film $[36,37]$ such that the external strain is purely due to the addition of the BTO capping layer. Magnetometry and magnetocaloric measurements were performed with a commercial (Quantum Design MPMS 3) superconducting quantum interference device (SQUID) magnetometer.

\section{RESULTS AND DISCUSSION}

X-ray diffraction (XRD) patterns [Fig. 1(a)] show epitaxial growth of both pseudocubic LCMO and tetragonal BTO with no impurity phases. The lattice constants of both films are within $0.2 \%$ of the bulk LCMO and BTO values indicating relaxed-epitaxy growth. This is a consequence of the large lattice misfit between $\mathrm{MgO}$ and $\mathrm{LCMO}(\sim 9 \%)$, which leads to an abrupt lattice relaxation through lattice defects $[36,37]$. The addition of these defects in the film can induce phase separation and broaden the transition, which can lead to the conversion of the first-order para-ferromagnetic transition into a secondorder transition. The distribution of defects can be qualitatively described by the temperature spread $(\Gamma)$ of the derivative of the resistivity $[32,38]$. We find $\Gamma \sim 15 \mathrm{~K}$ [Fig. 1(b)], which is larger than the previously reported [38] value $(\Gamma \sim 5 \mathrm{~K})$ for single crystalline LCMO. The extra broadening in the
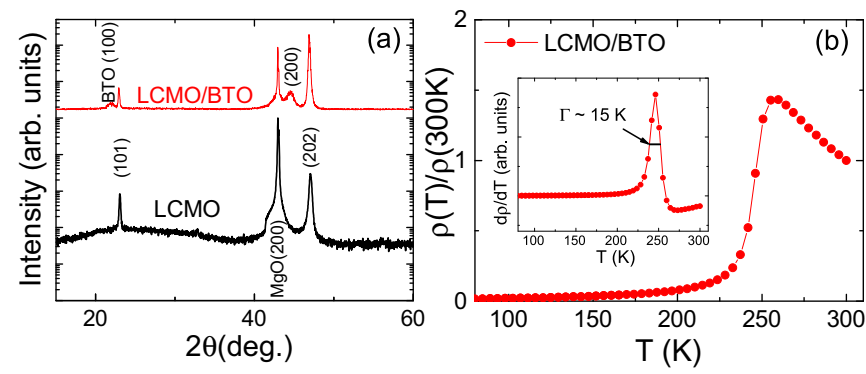

FIG. 1. (a) X-ray diffraction pattern of the LCMO film (bottom curve) and LCMO/BTO heterostructure (top curve). (b) Temperature dependent normalized resistivity measurements for LCMO/BTO bilayer and respective $d \rho / d T$ (inset).

transition temperature agrees with the expected large density of defects in the thin film and reduced transition temperature.

MCE and magnetization measurements [Figs. 2(a) and 2(b)] reveal a ferromagnetic to paramagnetic transition at $T_{C} \approx$ $250 \mathrm{~K}$ both for the plain LCMO film (green circles) as well as for the LCMO capped with BTO (blue triangles), confirming a "bulklike" behavior indicative of strain-relaxed films. The overall magnitude of the change in magnetic entropy $\left(\Delta S_{M}\right)$ at $T_{C}$ is approximately three times larger for the capped film $\left(1.48 \mathrm{~J} \mathrm{~kg}^{-1} \mathrm{~K}^{-1} \mathrm{~T}^{-1}\right)$ than that of the plain LCMO film $(0.5 \mathrm{~J}$ $\mathrm{kg}^{-1} \mathrm{~K}^{-1} \mathrm{~T}^{-1}$ ) seen in Fig. 2(a) and reported in the literature [14,32,39]. It has been previously shown [32] that the standard first-order magnetic phase transition of LCMO is transformed into a second-order phase transition in the limit of reduced dimensionality. To verify the order of the phase transformation in the LCMO/BTO bilayers, we analyzed the MCE data using two methods. First, we investigated the transition using a purely magnetic measurement approach based on the Banerjee criterion [40]. This criterion states that when a negative slope
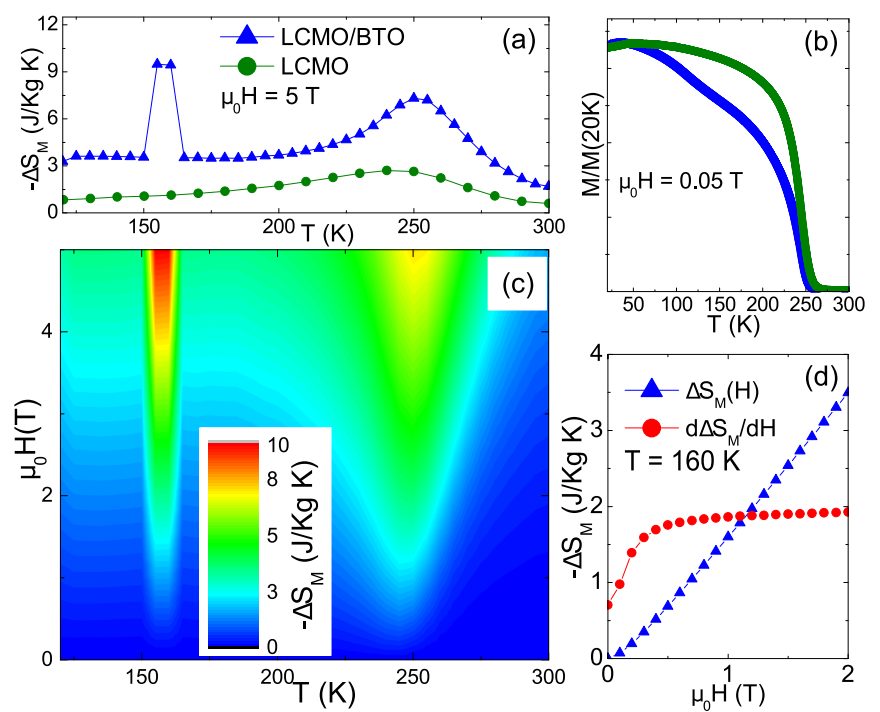

FIG. 2. (a) Magnetocaloric effect and (b) temperature dependent magnetization response of the LCMO/BTO bilayer and plain LCMO samples. (c) Magnetocaloric effect as a function of temperature and magnetic field. (d) Change in magnetic entropy and its derivative as a function of magnetic field. 

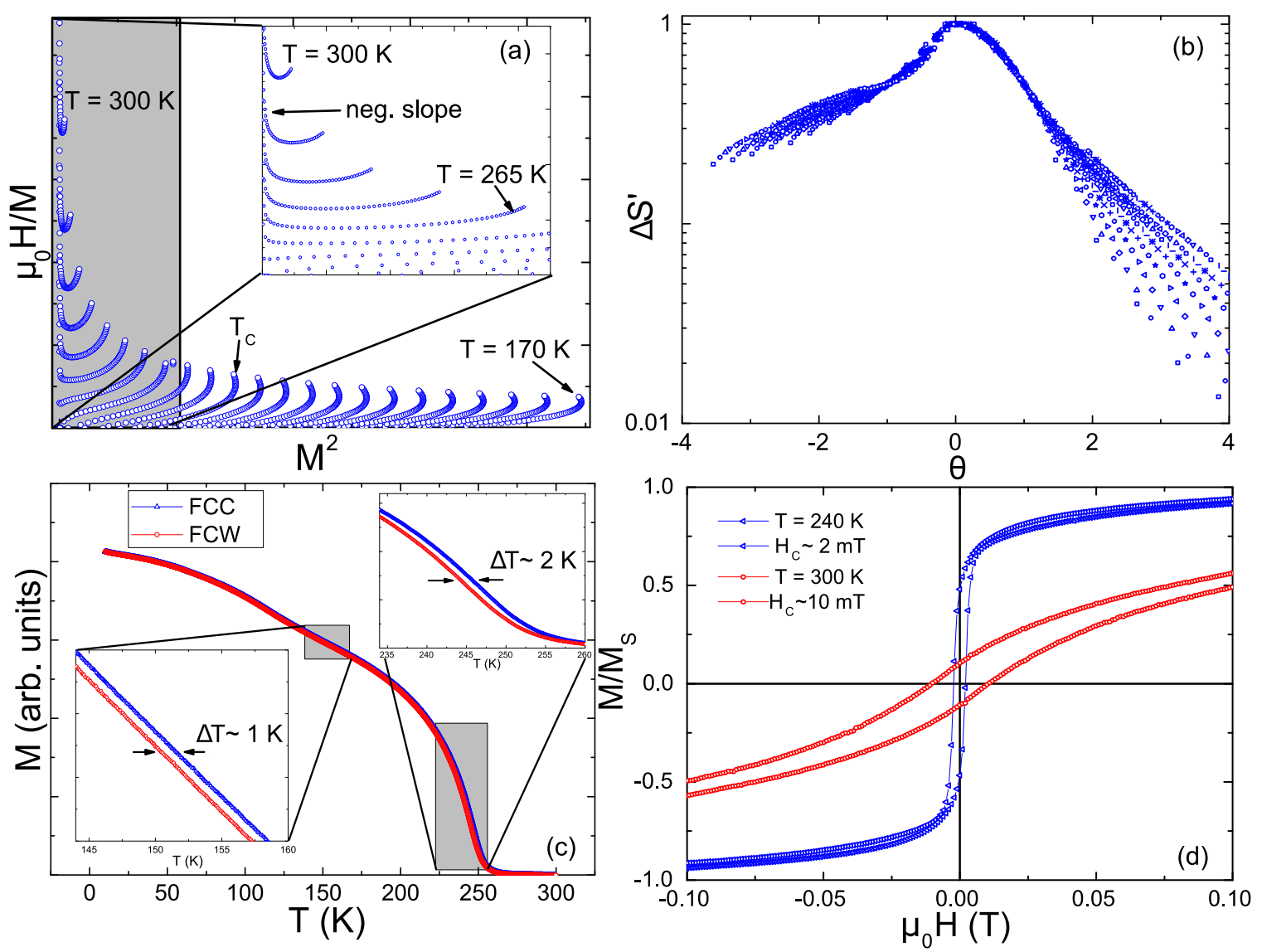

FIG. 3. (a) Isothermal Arrott-Belov curves above and below $T_{C}$. (b) Normalized magnetic entropy change near $T_{C}$. (c) Field-cooled cooling and field-cooled warming temperature dependent magnetization. (d) Field dependent magnetization at $300 \mathrm{~K}$ (red circles) and $240 \mathrm{~K}$ (blue triangles).

above $T_{C}$ occurs in the isothermal Arrott-Belov plots of $H / M$ versus $M^{2}$, the transition is of first order. Otherwise it is a second-order transition. From Fig. 3(a) one can see that above $T_{C}$ there is a negative slope (marked in the inset) indicative of a first-order transition. However, diamagnetic effects due to the substrate can influence this interpretation. Therefore, another test is required. We employed the relatively new method [41,42] based on rescaling $\Delta S_{M}$ curves such that they all collapse onto one single curve for various applied field $(H)$ values at a second-order phase transition. At a first-order phase transition, however, the underlying assumptions break down and there will be a slight spreading of the curves upon renormalization. Therefore, this is an ideal method for checking the order of magnetic transitions. The $\Delta S_{M}$ curves are normalized such that $\Delta S^{\prime}=\Delta S_{M} / \Delta S_{\text {Max }}$, where $\Delta S_{\text {Max }}$ is the peak value for each $\Delta S_{M}$ curve at each $H$ value. Then the temperature is renormalized as

$$
\theta= \begin{cases}-\left(T-T_{C}\right) /\left(T_{r 1}-T_{C}\right) & \text { if } T \leqslant T_{C}, \\ \left(T-T_{C}\right) /\left(T_{r 2}-T_{C}\right) & \text { if } T \geqslant T_{C}\end{cases}
$$

where the reference temperatures $\left(T_{r 1}, T_{r 2}\right)$ are chosen such that $\Delta S_{M}\left(T_{r 1}\right)=\Delta S_{M}\left(T_{r 2}\right)=\Delta S_{\text {Max }} / 2$. Clear separation in the curves, for $|\theta|>1$ [Fig. 3(b)] and a considerable increase in $\Delta S_{M}$ [Figs. 2(a) and 2(c)] indicate a first-order nature of the phase transition in the BTO capped film.
At $\approx 150 \mathrm{~K}, \mathrm{BTO}$ undergoes another structural transition from orthorhombic to rhombohedral, which is found to induce a giant change in both the magnetic entropy as well as the magnetization of the bilayer [Figs. 2(a) and 2(c)]. It is important to note that the magnitude of $\Delta S_{M}$ at $150 \mathrm{~K}\left(\approx 2 \mathrm{~J} \mathrm{~kg}^{-1} \mathrm{~K}^{-1} \mathrm{~T}^{-1}\right)$ is considerably reduced from the values reported for LCMO on BTO substrates $\left(\approx 9 \mathrm{~J} \mathrm{~kg}^{-1} \mathrm{~K}^{-1} \mathrm{~T}^{-1}\right)$ [14]. This is likely due to enhanced strain coupling between the clamped LCMO film and a single-crystalline BTO substrate. However, by straining LCMO from a cap layer, we provide an easier procedure for fabrication and tuning the magnetocaloric properties. At this transition temperature there is a sudden drop in the lattice parameters of BTO [43] that produces an interfacial change in the LCMO. This is compensated through the rest of the film by a sudden change in the magnetocrystalline anisotropy. The shift of the magnetic anisotropy can be compensated for by the application of a magnetic field of about $1 \mathrm{~T}$, thus leading to a saturation of $\partial \Delta S_{M} / \partial H$ [Fig. 2(d)]. Interestingly, in spite of the first-order nature of the transitions, there is minimal thermal and magnetic hysteresis [Figs. 3(c) and 3(d)] which is beneficial for magnetic refrigeration applications [33].

While information on the macroscopic magnetization can be gained from MCE measurements, interfacial properties are not easily decoupled from bulk properties, thus requiring a more local probe. It has been shown that colossal effects, such as CMR and colossal MCE, are enhanced when the 
material is in a phase-separated state, due to the rapid expansion of one phase that dominates over the other on application of a magnetic field [44-46]. Therefore, in order to fully understand phenomena induced at the interface, we employed x-ray absorption spectroscopy (XAS) combined with $\mathrm{x}$-ray magnetic circular dichroism (XMCD), and $\mathrm{x}$ ray linear dichroism (XLD). In particular XPEEM, based on the photoemission of electrons due to the absorption of $\mathrm{x}$ rays, offers spatially resolved element-specific XAS, XMCD, and XLD measurements. The small escape depth of the photoemitted electrons $(5-7 \mathrm{~nm})$ provides a means to investigate magnetic, structural, and electronic states at the interface between the two films.

The XAS images were normalized pixelwise to a pre-edge off-resonance image. XMCD and XLD images were calculated using the normalized XAS for circular right $\left(I_{R}\right)$, circular left $\left(I_{L}\right)$, linear horizontal $\left(I_{H}\right)$, and linear vertical $\left(I_{V}\right)$ polarized light and taking their difference over their sum $\left(I_{R}-I_{L}\right) /\left(I_{R}+I_{L}\right)$ and $\left(I_{H}-I_{V}\right) /\left(I_{H}+I_{V}\right)$, respectively. The data were obtained at the Ti $L_{2,3}$ edges and $\mathrm{Mn} L_{2,3}$ edges. XAS and XLD measurements were performed in the magnetically virgin state, while XMCD was measured at remanence after saturating the samples with a magnetic field.

There is a noticeable difference in the XAS between the plain LCMO (without the BTO cap) and the BTO capped films [Fig. 4(a)]. The XAS of the plain LCMO film (black circles) looks similar to that reported in the literature, where the peak at the $L_{3}$ edge $(642 \mathrm{eV})$ is associated with the Mn being in a mixture of $\mathrm{Mn}^{3+}$ and $\mathrm{Mn}^{4+}$ electronic states. The capped film, however, looks drastically different, with primary electronic contributions associated with $\mathrm{Mn}^{2+}$. It is well known that there is a tendency for formation of $\mathrm{Mn}^{2+}$ at a manganite interface [47]. In order ensure the $\mathrm{Mn}^{2+}$ valence state was not caused by prolonged exposure to air, the films were placed under vacuum after growth until the measurements were performed. As a
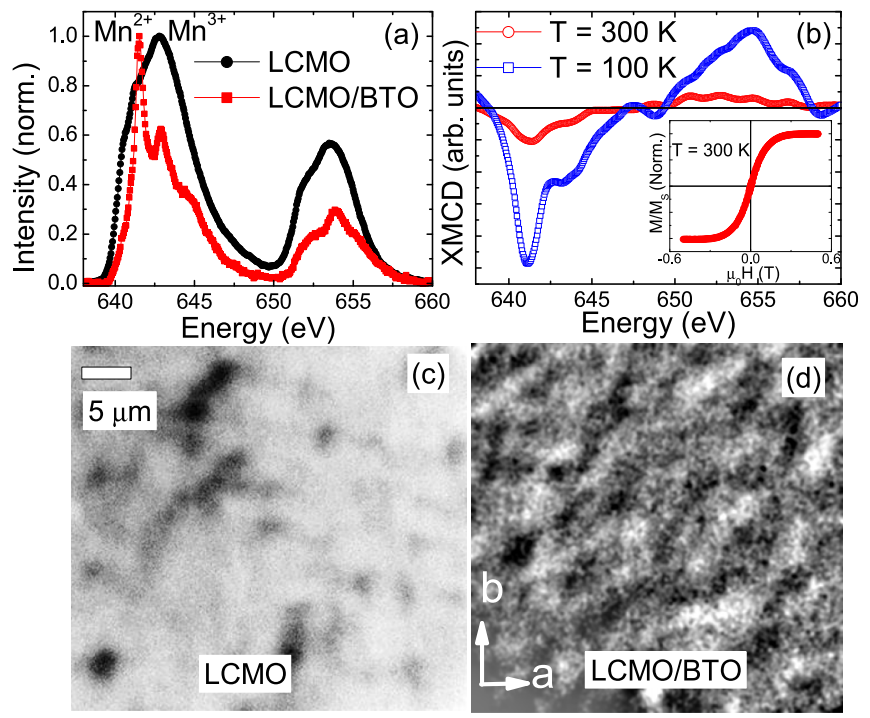

FIG. 4. (a) XAS of LCMO and LCMO with BTO cap at the Mn $L_{2,3}$ edge. (b) XMCD at both low temperature and room temperature for BTO capped LCMO and $M$ vs $H$ at $300 \mathrm{~K}$ for the capped film (inset). (c) and (d) XMCD images at $T=100 \mathrm{~K}$ and $E=642.5 \mathrm{eV}$ of LCMO and LCMO/BTO, respectively.
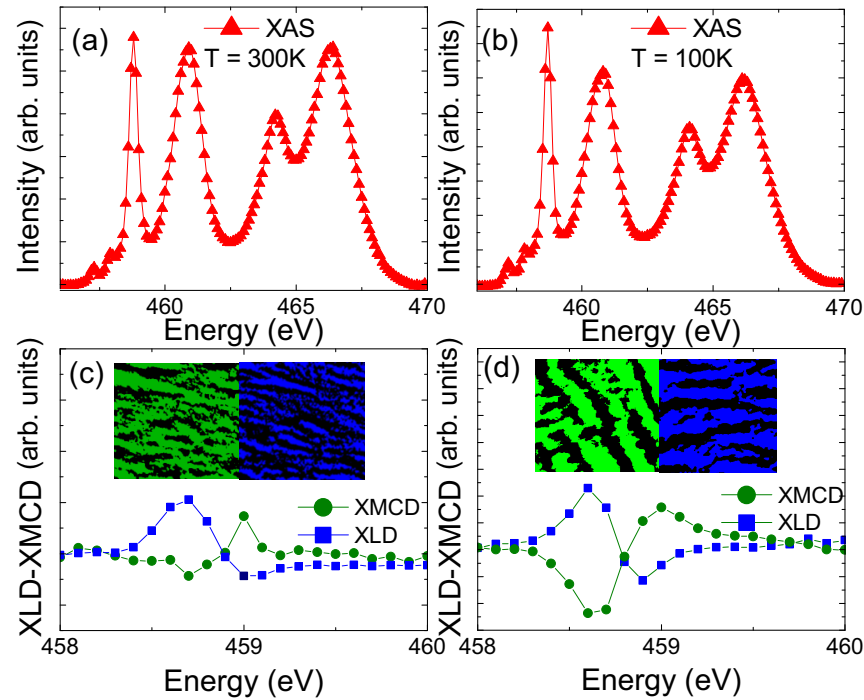

FIG. 5. (a) XAS for BTO/LCMO at (a) $300 \mathrm{~K}$ and (b) $100 \mathrm{~K}$. Linear and circular dichroism at the Ti $L_{2,3}$ edge for BTO/LCMO sample at (c) $300 \mathrm{~K}$ and (d) $100 \mathrm{~K}$. Insets are the autocorrelation of the XMCD (left panel) and XLD (right panel) partial maps at 300 and $100 \mathrm{~K}$, respectively.

further check, the films were also left in air for two months and, on second measurement, the results were identical, indicating that the altered electronic state is a result of electron doping at the interface. XMCD measurements were performed at 300 and $100 \mathrm{~K}$ [Fig. 4(b)], demonstrating that the bilayer sample is clearly ferromagnetic at room temperature, in agreement with the SQUID data [Fig. 4(b) inset]. XPEEM images taken at $\approx 100 \mathrm{~K}$ [Figs. 4(c) and 4(d)] reveal that LCMO exhibits a randomly oriented magnetic domain pattern, while the capped film appears to exhibit domains with preferential orientation along the BTO [110] crystal direction as predicted [16].

To investigate the electronic modifications further, XAS, XMCD, and XLD were also measured at the Ti $L_{2,3}$ edges [Figs. 5(a)-5(d)] both at room temperature and low temperature. The change in the relative intensities between the room temperature [Fig. 5(a)] and low temperature [Fig. 5(b)] measurements is indicative of the BTO structure transition [48]. At room temperature, we see a clear XMCD signal [Fig. 5(c)]. BTO typically exhibits the fundamentally nonmagnetic $\mathrm{Ti}^{4+}$ electronic configuration. However, since we observe a clear XMCD signal at both low and room temperatures, it follows that the interfacial $\mathrm{Ti}$ atoms are in a modified electronic state, leading to the formation of magnetic $\mathrm{Ti}^{3+}$. We note that the observation of magnetically polarized BTO in contact with a ferromagnetic system is now well documented in the literature $[15,49,50]$. However, since our films are not clamped to the substrate, we are able to investigate the physical properties on a more fundamental level. For this we performed XLD measurements [Figs. 5(c) and 5(d), blue squares], which is a crucial tool for the investigation of effects related to structural changes, e.g., orbit formation and ferroelectricity [51] by probing the distribution of empty Ti- $3 d$ states.

In order to quantify the orbital contribution to the x-ray signal we followed the procedure described in [52,53], and calculated the ratio of holes to electrons $(X)$ at the interface 

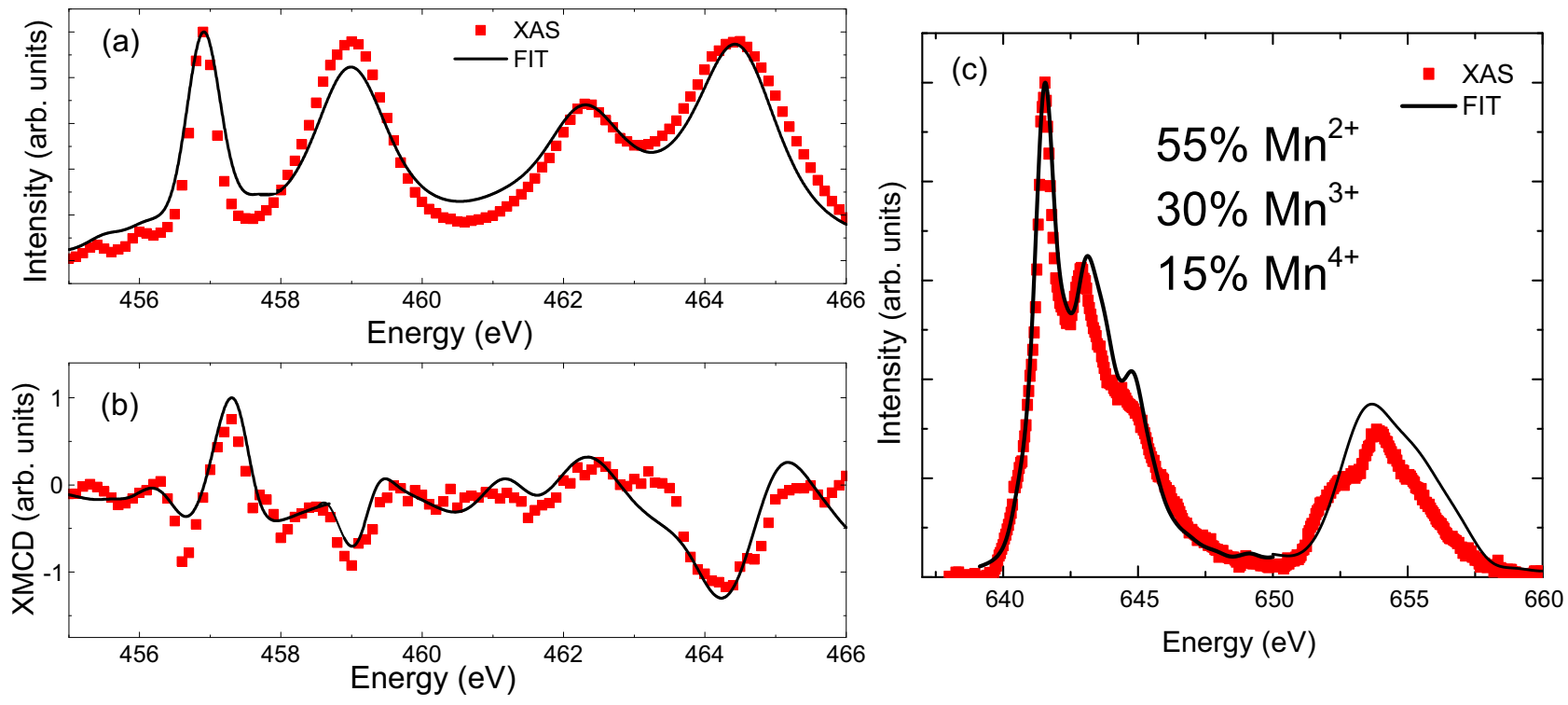

FIG. 6. (a) XAS and (b) XMCD experimental data (red squares) and fit calculated with CTM4XAS for LCMO with BTO cap at the Ti $L_{2,3}$ edge with $11 \% \mathrm{Ti}^{3+}$. (c) XAS experimental data and CTM4XAS calculated fit recorded at $\mathrm{Mn} L_{2,3}$ edge with $55 \% \mathrm{Mn}^{2+}$.

using

$$
X=\frac{3 \int_{L_{3,2}} I_{V}(E) d E}{4 \int_{L_{3,2}} I_{H}(E) d E-\int_{L_{2,3}} I_{V}(E) d E},
$$

where $I_{V}$ and $I_{H}$ are the geometrically corrected XAS intensity for the X-ray polarization parallel and perpendicular to the [001] axis of the film, respectively. For a material with predominantly hole doping, $X>1$, and for electron doping, $X<1$. For the capped films, we find that the ratio is $X=0.8$ corresponding to a surplus of electrons at the interface. The electron doping could be due to oxygen vacancies in the LCMO; however, this would result in a larger unit cell which is not evident in the XRD patterns [Fig. 1(a)]. Therefore, the electron doping at the interface is likely due to hybridization between $\mathrm{Mn}^{3+/ 4+}$ and $\mathrm{Ti}^{4+}$ electronic states. This results in an enhancement of the $\mathrm{Mn}^{2+/ 3+}$ and $\mathrm{Ti}^{3+}$ electronic structure ratio at the interface.

We used the CTM4XAS program [54] to further analyze the $\mathrm{Mn}$ and Ti electron valency deduced from XAS/XMCD data in Figs. 4(a), 5(a) and 5(c), respectively. Starting with the Ti $L_{2,3}$ edges, we first simulated the XAS with $\mathrm{Ti}^{4+}$ tetragonal symmetry using values from previous studies [55] [Fig. 6(a)]. The experimental peaks are broader than the simulated peaks due to hybridization effects and are dealt with by Lorentz broadening each peak individually. The simulated results for the pure $\mathrm{Ti}^{4+}$ exhibits no XMCD signal, as expected. However, with the addition of $11 \% \mathrm{Ti}^{3+}$ contribution in the simulations we were able to reproduce the XMCD signal [Fig. 6(b)]. Differentiating between $\mathrm{Ti}^{3+}$ and $\mathrm{Ti}^{4+}$ is not trivial, since the interfacial electronic states exhibit mixed $\mathrm{Ti}$ and Mn valency [56] and hybridization between Mn-O-Ti. Nevertheless, we have shown strong evidence for a surplus of electrons at the interface between the two materials likely leading to the formation of nanoscale ferromagnetic and ferroelectric clusters giving rise to the enhancement of the magnetic and electronic properties at the interface. We have also simulated the Mn $L$ edge [Fig. 6(c)] and found that the dominant contribution $(55 \%)$ is due to $\mathrm{Mn}^{2+}$, whereas the remaining $45 \%$ belongs to a nominal mixture of $\mathrm{Mn}^{3+/ 4+}$ at $30 \%$ and $15 \%$, respectively. It is important to note that the ratio of $\mathrm{Mn}^{3+}$ to $\mathrm{Mn}^{4+}$ remains consistent with the expected value for this LCMO stoichiometry.

\section{CONCLUSIONS}

In summary, using PEEM, MCE, and magnetometry measurements, we have shown that at epitaxial interfaces between BTO and LCMO there are modifications to the electronic configurations of both the LCMO and BTO. As a result of the interfacial coupling between the two materials there are small scale ferromagnetic correlations above $T_{C}$ leading to room temperature magnetic ordering. The addition of the BTO capping layer also leads to large MCE at $150 \mathrm{~K}$ and an enhancement of the MCE at $250 \mathrm{~K}$ due to the orthorhombicrhombohedral and orthorhombic-tetragonal structural phase transitions in BTO, respectively. By enhancing the MCE via thin-film heterostructures rather than substrate induced effects, we obtained a considerable decrease in magnetic and thermal hysteresis. We have therefore demonstrated a method of strain engineering that enhances the MCE properties. This is a flexible approach that may be applied to enhance and control further phenomena in complex oxide heterostructures such as superconductivity and multiferroicity.

\section{ACKNOWLEDGMENTS}

The authors thank Michele Buzzi and Victorino Franco for assistance with measurements and fruitful discussions. Part of this work was performed at the SIM beamline of the Swiss Light Source, Paul Scherrer Institut, Switzerland. A.K.S. acknowledges funding from the European Community's Seventh Framework Program (FP7/2007-2013) under Grant Agreement No. 290605 (COFUND: PSIFELLOW). 
[1] G. H. Jonker and J. H. V. Stanten, Physica 16, 337 (1950).

[2] Y. Tokura and Y. Tomioka, J. Magn. Magn. Mater. 200, 1 (1999).

[3] S. Jin et al., Science 264, 413 (1994).

[4] T. Kimura et al., Nature (London) 426, 55 (2003).

[5] H. Kuwahara et al., Science 270, 961 (1995).

[6] J. M. D. Coey, M. Viret, L. Ranno, and K. Ounadjela, Phys. Rev. Lett. 75, 3910 (1995).

[7] M. B. Salamon and M. Jaime, Rev. Mod. Phys. 73, 583 (2001).

[8] C. Zener, Phys. Rev. 82, 403 (1951).

[9] A. J. Millis, R. Mueller, and B. I. Shraiman, Phys. Rev. B 54, 5389 (1996).

[10] A. J. Millis, R. Mueller, and B. I. Shraiman, Phys. Rev. B 54, 5405 (1996).

[11] A. Moreo et al., Science 283, 2034 (1999).

[12] A. J. Millis, P. B. Littlewood, and B. I. Shraiman, Phys. Rev. Lett. 74, 5144 (1995).

[13] H. Röder, J. Zang, and A. R. Bishop, Phys. Rev. Lett. 76, 1356 (1996).

[14] X. Moya et al., Nat. Mater. 12, 52 (2013).

[15] W. Eerenstein et al., Nat. Mater. 6, 348 (2007).

[16] J. Paul, T. Nishimatsu, Y. Kawazoe, and U. V. Waghmare, Phys. Rev. Lett. 99, 077601 (2007).

[17] Y. K. Yoo et al., Nature (London) 406, 704 (2000).

[18] K. H. Ahn, T. Lookman, and A. R. Bishop, Nature (London) 428, 401 (2004).

[19] K. Lai et al., Science 329, 190 (2010).

[20] P. Orgiani et al., Phys. Rev. B 82, 205122 (2010).

[21] C. A. F. Vaz, J. A. Moyer, D. A. Arena, C. H. Ahn, and V. E. Henrich, Phys. Rev. B 90, 024414 (2014).

[22] C. A. F. Vaz et al., J. Phys.: Condens. Matter 24, 333201 (2012).

[23] P. Zubko et al., Annu. Rev. Condens. Matter Phys. 2, 141 (2011).

[24] E. Jiménez, J. Camarero, J. Sort, J. Nogués, N. Mikuszeit, J. M. García-Martín, A. Hoffmann, B. Dieny, and R. Miranda, Phys. Rev. B 80, 014415 (2009).

[25] T. S. Santos, B. J. Kirby, S. Kumar, S. J. May, J. A. Borchers, B. B. Maranville, J. Zarestky, S. G. E. te Velthuis, J. van den Brink, and A. Bhattacharya, Phys. Rev. Lett. 107, 167202 (2011).

[26] C. A. F. Vaz et al., Adv. Mater. 22, 2900 (2010).

[27] M. Nagao et al., Nat. Nanotechnol. 8, 325 (2013)

[28] L. Li et al., Nat. Phys. 7, 762 (2011).

[29] M. Bibes et al., Adv. Phys. 60, 5 (2011).
[30] S. Fusil et al., Annu. Rev. Mater. Res. 44, 91 (2014).

[31] M.-H. Phan and S.-C. Yu, J. Magn. Magn. Mater. 308, 325 (2007).

[32] P. Lampen et al., Appl. Phys. Lett. 102, 062414 (2013).

[33] N. S. Bingham et al., J. Appl. Phys. 106, 023909 (2009).

[34] P. Lampen, N. S. Bingham, M. H. Phan, H. Srikanth, H. T. Yi, and S. W. Cheong, Phys. Rev. B 89, 144414 (2013).

[35] V. Franco, A. Conde, J. M. Romero-Enrique, and J. S. Blázquez, J. Phys.: Condens. Matter 20, 285207 (2008).

[36] E. Gommert et al., J. Appl. Phys. 85, 5417 (1999).

[37] S. R. Singamaneni et al., Appl. Phys. Rev. 3, 031301 (2016).

[38] M. Egilmez, K. H. Chow, and J. Jung, Appl. Phys. Lett. 92, 162515 (2008).

[39] X. Zhang et al., Appl. Phys. Lett. 69, 3596 (1996).

[40] B. K. Banerjee, Phys. Lett. 12, 16 (1964).

[41] C. M. Bonilla et al., J. Appl. Phys. 107, 09E131 (2010).

[42] V. Franco, J. S. Blázquez, and A. Conde, Appl. Phys. Lett. 89, 222512 (2006).

[43] H. Terashita, B. Myer, and J. J. Neumeier, Phys. Rev. B 72, 132415 (2005)

[44] M. H. Phan, M. B. Morales, N. S. Bingham, H. Srikanth, C. L. Zhang, and S. W. Cheong, Phys. Rev. B 81, 094413 (2010).

[45] N. S. Bingham, P. Lampen, M. H. Phan, T. D. Hoang, H. D. Chinh, C. L. Zhang, S. W. Cheong, and H. Srikanth, Phys. Rev. B 86, 064420 (2012).

[46] A. Alberca et al., Sci. Rep. 5, 17926 (2015).

[47] S. Valencia, A. Gaupp, W. Gudat, Ll. Abad, Ll. Balcells, A. Cavallaro, B. Martinez, and F. J. Palomares, Phys. Rev. B 73, 104402 (2006)

[48] M. Bugnet, G. Radtke, S. Y. Woo, G.-Z. Zhu, and G. A. Botton, Phys. Rev. B 93, 020102(R) (2016).

[49] S. M. Wu et al., Nat. Mater. 9, 756 (2010).

[50] C.-G. Duan, S. S. Jaswal, and E. Y. Tsymbal, Phys. Rev. Lett. 97, 047201 (2006).

[51] R. V. Chopdekar et al., Phys. Rev. B 86, 014408 (2012).

[52] C. Schmitz-Antoniak et al., Nat. Commun. 4, 2051 (2013).

[53] M. Wu et al., Phys. Rev. B 88, 125124 (2013).

[54] E. Stavitski and F. M. F. de Groot, Micron 41, 687 (2010).

[55] A. Chassé, S. Borek, K.-M. Schindler, M. Trautmann, M. Huth, F. Steudel, L. Makhova, J. Gräfe, and R. Denecke, Phys. Rev. B 84, 195135 (2011).

[56] M. Salluzzo et al., Phys. Rev. Lett. 102, 166804 (2009). 